\title{
Uso de antimuscarínicos en pacientes con STUI-HBP y sintomas de VHA: maestría clínica y resistencia a perder el miedo al riesgo de retención urinaria
}

\author{
Ruiz Cerdá JL. \\ Servicio de Urología. Hospital Universitario La Fe. Valencia. \\ Actas Urol Esp. 2008;32(10):957-960
}

$\mathrm{E}^{1}$ 1 uso de antimuscarínicos en pacientes con HBP y sintomatología del tracto urinario inferior compatible con vejiga hiperactiva (VHA) es un tema que hemos discutido en ocasiones anteriores ${ }^{1}$ y que continúa generando controversia. Principalmente, porque la evidencia científica disponible no parece ser suficiente para superar el temor a provocar retención urinaria tras la toma prolongada de antimuscarínicos en pacientes con obstrucción uretral.

Son varias las razones que llevan a considerar el uso de antimuscarínicos en esta población de pacientes, entre ellas: el incremento de la prevalencia de los sintomas de VHA en el hombre ${ }^{2,3}$ (sobre todo en las últimas décadas de la vida cuando la HBP también es más prevalente), el solapamiento con los síntomas del tracto urinario inferior relacionados con HBP (STUI-HBP) correspondientes a la fase de llenado (que afectan más a la calidad de vida que los de vaciado) ${ }^{4}, y$, por último, el hecho de que los fármacos para el tratamiento de pacientes con STUI-HBP (alfabloqueantes, inhibidores de las 5 alfa reductasa) actúan únicamente sobre el componente obstructivo (uretral) y no sobre el componente vesical, lo que hace que se cubran parcialmente las expectativas de mejora sintomatológica de los pacientes.

Por otra parte, también puede justificarse el uso de antimuscarínicos ante la presencia de HBP, obstrucción y STUI desde el punto de vista fisiopatológico, pues aunque tradicionalmente se ha establecido una relación de causalidad entre obstrucción por HBP y desarrollo de hiperactividad del detrusor, la evidencia clínica ha puesto de manifiesto que la desobstrucción no necesariamente hace desaparecer la hiperactividad ${ }^{5}$. Incluso, aunque desaparezca, si el paciente es seguido a largo plazo, la hiperactividad reaparece ${ }^{5}$.
Aunque en la actualidad exista suficiente evidencia científica para afirmar que el riesgo a provocar retención urinaria con la administración de antimuscarínicos en este grupo de pacientes es mínimo a las dosis utilizadas en la práctica clínica habitual ${ }^{6,7}$, los datos disponibles provienen de un número limitado de ensayos clínicos que para muchos urólogos no tienen el suficiente peso para respaldar este tratamiento.

\section{MAESTRÍA CLÍNICA INDIVIDUAL}

Puestas así las cosas, la pregunta que cabe plantearse es la siguiente: ¿la escasa evidencia científica invalida el uso de antimuscarínicos en estos pacientes? o lo que es lo mismo ¿es posible seguir tomando decisiones individuales aunque tengamos un escaso soporte científico? Mi respuesta es afirmativa. Siempre que el urólogo sea capaz de utilizar su maestría clínica individual, entendiendo como maestría clínica individual "el dominio del conocimiento y el juicio que los clínicos individuales adquieren a través de su experiencia clínica y de la práctica clínica”. Esta definición, cuyo autor es el padre de la medicina basada en la evidencia, David Sackett, significa realizar un diagnóstico más efectivo y más eficiente e identificar y utilizar de forma más sensible los problemas, derechos y preferencias de los pacientes ${ }^{8}$.

Desde mi punto de vista, tomar decisiones de diagnostico y tratamiento en este grupo de pacientes basadas en la maestría clínica individual está totalmente justificado. En primer lugar, porque los síntomas de llenado son los más preocupantes para el enfermo y los que más afectan a su calidad de vida. En segundo lugar, porque la seguridad de los fármacos antimuscarínicos está bien documentada. Por último, el probable riesgo de retención crónica o aguda es totalmente reversible y no compromete la vida del paciente. Sobre todo si se tiene un seguimiento estrecho y cuidadoso. 
Según mi opinión, son dos los aspectos fundamentales para manejar a este grupo de pacientes: su espectro sintomático y las exploraciones complementarias disponibles en nuestro ámbito de trabajo para el diagnóstico funcional correcto del tracto urinario inferior.

\section{INFORMACIÓN DESDE LA PRÁCTICA CLÍNICA}

Puesto que se trata de un tema en el que se ponen en cuestión conocimientos y conceptos muy arraigados en una patología de máximo interés para el urólogo como es la HBP, y la evidencia científica no es abundante, es de interés conocer cómo los urólogos están manejando a estos pacientes. En otras palabras, como están llevando a cabo su maestría clínica individual. Para ello, posteriormente a la publicación de mi artículo y con la ayuda de Laboratorios Almirall S.A., se llevaron a cabo una serie de sesiones para captar la opinión de 114 urólogos sobre aspectos relacionados con el manejo diagnóstico y terapéutico de pacientes con STUIHBP y VHA. Las opiniones se recogieron siguiendo el método Metaplan, ya explicado anteriormente ${ }^{9}, \mathrm{y}$ siendo representativas de la geografía española. El perfil de urólogos consultados fue: 10 mujeres (8\%) y 104 hombres (92\%), con un promedio de edad de 47 años. En cuanto a su perfil laboral, principalmente desarrollaban su actividad en hospitales (62\%), aunque también en el ámbito de la asistencia ambulatoria y la consulta privada (menos del 20\%).

El objetivo principal de las sesiones era obtener información sobre la práctica clínica habitual de los urólogos respecto a la frecuencia con que se presenta este tipo de pacientes, como se diagnostican, cuando se llega al diagnostico de $\mathrm{VH}$ y las indicaciones para el uso de antimuscarínicos así como el perfil de los pacientes.

Los urólogos consideran que los pacientes con STUI-HBP y VHA representan un grupo pequeño. Sólo el 9\% de los consultados los ve de manera muy frecuente en su práctica clínica habitual. Porcentaje relativamente bajo si lo comparamos con el 91\% de la VH (Fig. 1). Desde el punto de vista cualitativo, la asociación de VH con la HBP es aceptada por la mayoría de los urólogos consultados, aunque con un grado diferente de percepción. Existen dos posturas básicas que son: los que entienden que puede tratarse de patologías/síndromes independientes y los creen que la VH se asocia siempre a la HBP.

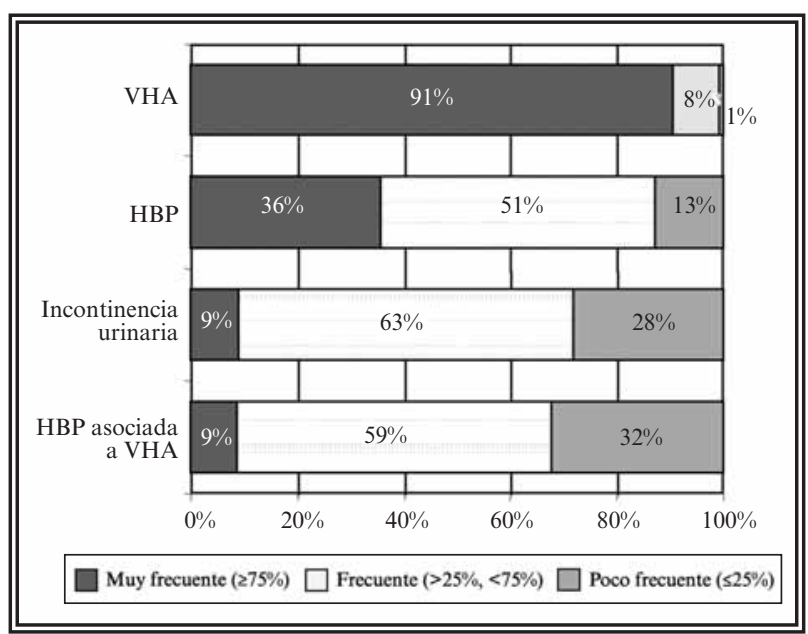

FIGURA 1. Frecuencia de VHA y de HBP en la práctica clinica habitual urológica.

En cuanto al manejo diagnóstico, se basa fundamentalmente en la determinación del tamaño prostático, mediante tacto rectal o ecografía, y estudio flujométrico. La utilización real del cuestionario sintomatológico IPSS es muy baja. El 70\% no utiliza nunca el cuestionario completo ni tampoco es cumplimentado por el paciente. Lo que hacen en la consulta es utilizar algunas de sus preguntas durante la anamnesis dirigida. Por lo tanto, según los datos obtenidos (Fig. 2), el 90\% de los urólogos toman sus decisiones únicamente teniendo en cuenta el tamaño prostático y la información subjetiva de síntomas del paciente recogida de forma no sistematizada. El $67 \%$ se ayuda de la información aportada por la flujometría. La mayoría (82\%) nunca realiza estudios de presión y flujo. La razón fundamental es su falta de disponibilidad.

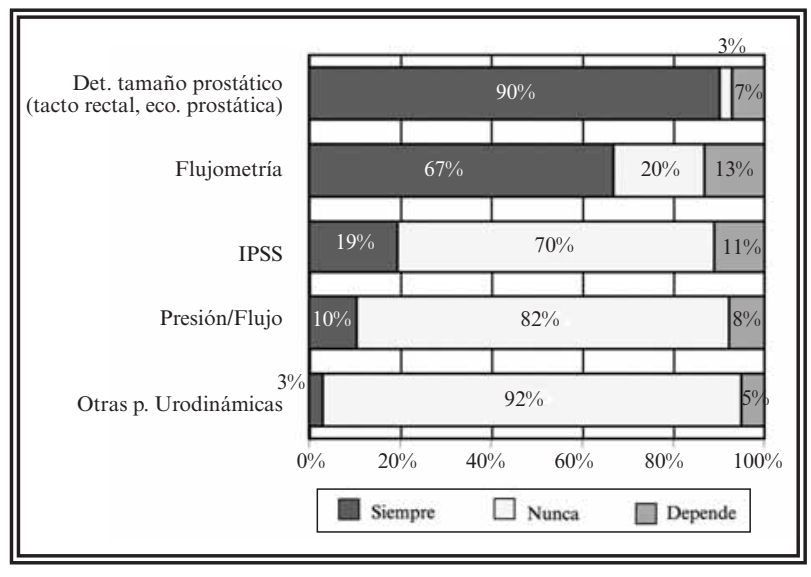

FIGURA 2. Frecuencia en el uso de técnicas diagnósticas en pacientes con sintomas irritativos y/o obstructivos. 
Un aspecto esencial para el diagnóstico de cualquier enfermedad o síndrome es pensar en él. Porque no se puede diagnosticar lo que no se conoce. El 38\% de los consultados consideran razonable pensar que se encuentran ante un paciente con STUI-HBP y síntomas de VHA sólo cuando existe un predominio de los sintomas irritativos y el 19\% únicamente ante el fracaso del tratamiento con alfabloqueantes (Fig. 3).

\begin{tabular}{||l|c|c||}
\hline \multicolumn{1}{|l||}{} & $\%$ & $\%$ \\
\hline Predominio de síntomas de llenado/irritativos & 38 & 48 \\
\hline Escasa respuesta al tratamiento con $\alpha$-bloqueantes & 19 & 24 \\
\hline Trastornos neurológicos & 10 & 13 \\
\hline Descartada una obstrucción & 7 & 9 \\
\hline Por descarte de otras patologías con síntomas similares & 6 & 7 \\
\hline En todos & 6 & 7 \\
\hline En caso de enfermedades concomitantes & 5 & 6 \\
\hline Cuando las pruebas lo indiquen & 5 & 6 \\
\hline Síntomas obstructivos poco evidentes con flujometría normal & 4 & 5 \\
\hline
\end{tabular}

FIGURA 3. Tipología de pacientes donde se plantea la posibilidad de una VHA.

De hecho, el perfil de los pacientes en los que se piensa que un antimuscarínico podría ser beneficioso, lo constituyen estas dos condiciones, 36\% y 19 respectivamente. Aunque, la principal condición es la ausencia de obstrucción con un $47 \%$.

El grado de obstrucción merece un comentario aparte. Como se muestra en la Figura 4, los urólogos consultados consideran razonable el tratamiento con antimuscarínicos principalmente en los casos de obstrucción leve. Un 60\% consideraría su uso muy razonable (21\%) o razonable (39\%). Sólo un 30\% lo consideraría razonable en caso de obstrucción moderada.

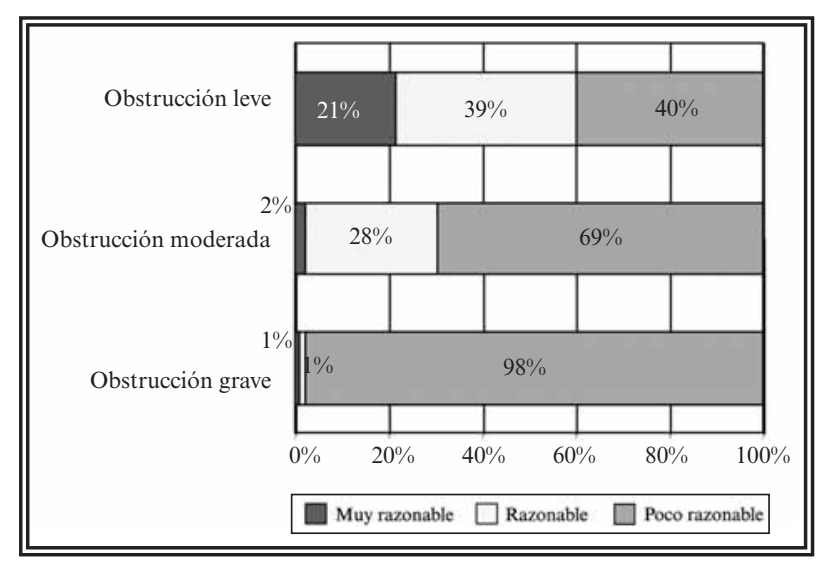

FIGURA 4. Porcentaje de uso de antimuscarínicos en relación al grado de obstrucción vesical.

\section{RESUMEN Y CONCLUSIONES}

Por lo tanto, y de acuerdo a estos datos se pueden extraen las siguientes conclusiones:

A pesar de los estudios epidemiológicos llevados a cabo sobre VHA y su amplia difusión, sigue existiendo una falta de conocimiento sobre las tasas de prevalencia de VHA en hombres. Un porcentaje bajo de urólogos piensan en que los pacientes con HBP puedan tener una VHA asociada. Únicamente piensa en esta asociación cuando existe un predominio de síntomas de llenado o escasa respuesta al tratamiento con alfabloqueantes.

Por lo general, en su práctica habitual, el porcentaje de urólogos que considera razonable utilizar antimuscarínicos en éste grupo de pacientes es francamente bajo. La justificación principal es la falta de estudios científicos que avalen su utilización. Todo ello, a pesar de que cada día hay más evidencia sobre seguridad y eficacia de los antimuscarínicos en este grupo de pacientes ${ }^{10}$, sigue existiendo precaución excesiva al uso de antimuscarínicos en este grupo de pacientes. El miedo a la retención supera al beneficio que se podría obtener tratando los síntomas de VHA. La obstrucción uretral sigue siendo el mayor condicionante a la hora de plantearse la utilización de antimuscarínicos. Su uso estaría justificado sólo en caso de obstrucción leve o como tratamiento de segunda línea ante el fracaso de alfabloqueantes.

La utilización de antimuscarínicos se realiza en base a la maestría individual, pero sorprende que sólo un 10\% utilice los estudios de presión y flujo, única exploración urodinámica que permite objetivar y cuantificar el grado de obstrucción e indicar el tratamiento más seguro y adecuado desde el punto de vista fisiopatológico.

\section{REFERENCIAS}

1. Ruiz Cerdá JL. El uso de antimuscarínicos en pacientes varones con síntomas del tracto urinario inferior por hiperplasia benigna de próstata y síntomas de vejiga hiperactiva. Actas Urol Esp. 2006;30(9):849-855.

2. Milsom I, Abrams P, Cardozo L, Roberts RG, Thüroff J, Wein AJ. How widespread are the symptoms of an overactive bladder and how are they managed? A population-based prevalence study. BJU International. 2001 Jun;87 (9):760-766.

3. Stewart WF, Van Rooyen JB, Cundiff GW, Abrams P, Herzog AR, Corey R et al. Prevalence and burden of overactive bladder in the United States. World J Urol. 2003 May;20(6):327336. 
4. Irwin DE, Milsom I, Reilly K, Hunskaar S, Kopp Z, Herschorn S et al. Prevalence of overactive bladder syndrome: European results from the EPIC study. Eur Urol. Suppl 2006;5(2):115.

5. Thomas AW, Cannon A, Bartlett E, Jones J, Abrams P. The natural history of lower urinary tract dysfunction in men: minimum 10-year urodynamyc follow-up of untreated bladder obstruction. BJU Int. 2005 Dec;96(9):1301-1306.

6. Andersson KE, Yoshida M. Antimuscarinics and the over active detrusor - which is the main mechanism of action? Eur Urol. 2003 Jan;43(1): 1-5.

7. Reynard JM. Does anticholinergic medication have a role for men with lower urinary tract symptoms/benig prostatic hyperplasia either alone or in combination with other agents? Curr Opin Urol. 2004 Jan;14(1):13-16.

8. Sackett D, Roseberg WM, Muir Gray JA, Haynes RB Richardson WS Evidence based medicine: What it is and what it isn't. [Editorial] BMJ. 1996;312(7):1-72.
9. Ruiz Cerdá JL, Arlandis Gúzman S, Trassierra Villa M, García Fadrique G, Morales Solchaga G, Jiménez Cruz JF. Análisis de la adherencia de los urólogos españoles a las recomendaciones de diagnóstico y tratamiento de las guías de práctica clínica sobre incontinencia urinaria. Actas Urol Esp. 2007;31(10):1148-1160.

10. Kaplan SA, Roehrborn CG, Chancellor M, Carlsson M, Bavendam T, Guan Z. Extended-release tolterodine with or without tamsulosin in men with lower urinary tract symptoms and overactive bladder: effects on urinary symptoms assessed by the International Prostate Symptom Score. BJU Int. 2008 May 26. [Epub ahead of print].

Correspondencia autor: Dr. J.L. Ruiz Cerdá

Servicio de Urología. Hospital Universitario La Fe

Avda. Campanar, 21 - 46009 Valencia. Tel.: 963862700

E-mail autor: Jose.L.Ruiz@uv.es

Información artículo: Editorial

Trabajo recibido: julio 2008 